Full paper

\title{
Hyper-stretchable self-powered sensors based on electrohydrodynamically printed, self-similar piezoelectric nano/microfibers
}

\author{
YongAn Huang ${ }^{\mathrm{a}, \mathrm{b}}$, Yajiang Ding ${ }^{\mathrm{a}, \mathrm{b}}$, Jing Bian ${ }^{\mathrm{a}, \mathrm{b}}$, Yewang $\mathrm{Su}^{\mathrm{c}}$, Jun Zhou ${ }^{\mathrm{d}}$, Yongqing Duan ${ }^{\mathrm{a}, \mathrm{b}, *}$, \\ Zhouping Yin ${ }^{\mathrm{a}, \mathrm{b}, *}$ \\ a State Key Laboratory of Digital Manufacturing Equipment and Technology, Huazhong University of Science and Technology, Wuhan 430074, China \\ ${ }^{\mathrm{b}}$ Flexible Electronics Research Center, Huazhong University of Science and Technology, Wuhan 430074, China \\ ' State Key Laboratory of Nonlinear Mechanics, Institute of Mechanics, Chinese Academy of Sciences, Beijing 100190, China \\ d Wuhan National Laboratory for Optoelectronics and School of Optical and Electronic Information, Huazhong University of Science and Technology, Wuhan 430074, \\ China
}

\section{A R T I C L E I N F O}

\section{Keywords:}

Stretchable electronics

Self-powered sensor

Electrohydrodynamic printing

Piezoelectric nanofiber

Buckling mechanics

\begin{abstract}
A B S T R A C T
Hyper-stretchable self-powered sensors with high sensitivity and excellent stability using low-cost, printable, organic nanomaterials are attractive for immense applications. Here we present self-similar piezoelectric nano/ microfibers for a hyper-stretchable self-powered sensor that demonstrates high stretchability $(>300 \%)$, low detection limit $(0.2 \mathrm{mg}$ ), and excellent durability ( $>1400$ times at strain $150 \%$ ). A proposed helix electrohydrodynamic printing technique (HE-Printing) in combination with in-surface self-organized buckling is used to fabricate aligned self-similar poly[vinylidene fluoride] (PVDF) nano/microfibers with in situ mechanical stretch and electrical poling to produce excellent piezoelectric properties. The hyper-stretchable self-powered sensors have shown repeatable and consistent electrical outputs with detection limit an order of magnitude smaller than the other stretchable sensors. Additionally, such sensors can simultaneously measure the own status and the extra multiply physical quantities, such as lateral pressure, impulse rate and applied strain. The high sensitivity can be further utilized to remotely detect human motion in addition to sensing a piece of paper with $1 \mathrm{~mm} \times 1 \mathrm{~mm}$. Further the fiber-based sensors can avoid the catastrophic collapse or wrinkling of serpentine film-based structure during stretching.
\end{abstract}

\section{Introduction}

Stretchable sensor is a field that is rapidly advancing as driven by immense applications including wearable and epidermal electronics [1-5], human-machine interface [6,7], rehabilitation and health monitoring [8], and e-skin [9-12] due to their conformability to curved surfaces and ability to withstand high strain levels. The major success is the judicious and specialized research of the fabrication processes and device designs of highly stretchable architecture. The stretchability has been achieved by utilizing nanomaterials [13-15], rubberlike materials $[16,17]$, liquid metal and conductive liquid $[18,19]$, and serpentine/ self-similar/kirigami/bioinspired patterns [20-24]. However, such sensors are commonly fabricated by photolithographic or spin-coating techniques that are either complicated and expensive or incompatible with large-scale, low-cost fabrication. Usually, the inkjet printing and direct-writing techniques were normally suitable for flexible, not stretchable, devices [25-27]. It is significant to develop low-cost printing routes of high-performance, highly stretchable sensors.
In the past decade, the development of stretchable sensors achieved great progresses, including the stretchability, sensitivity, and fabrication $[21,28,29]$. Many stretchable sensors based on piezoresistive effect were reported, which even has the ability to detect strain up to $280 \%$ [13]. However, an external power supply to generate bias voltage was inevitable for these stretchable sensors during the operation and characterization, otherwise none of them can normally work [30,31]. It would increase the device cost and structural complexity considering power consumption. A reported film-based self-powered piezoelectric system could be uniaxially stretched as high as $30 \%$ via the serpentine metal lines, however the sensing units were still unstretchable [32]. Stretchable self-powered sensor was introduced by virtue of helix fibers, and the stretchability achieve 25\% [33]. Further, a fiber-convolved layout tremendously enlarged the stretchability up to $70 \%$ [2]. When the sensors can be stretched further to larger than $100 \%$ or even $300 \%$, and have low detection limit, the applications can tremendously expand.

Here, we proposed self-similar piezoelectric nano/microfibers for a

\footnotetext{
* Corresponding authors at: State Key Laboratory of Digital Manufacturing Equipment and Technology, Huazhong University of Science and Technology, Wuhan 430074, China.

E-mail addresses: duanyongqing@hust.edu.cn (Y. Duan), yinzhp@mail.hust.edu.cn (Z. Yin).
} 
hyper-stretchable self-powered sensor (HSS) which demonstrated ultrahigh mechanical stretchability ( $>300 \%$ ), ultra-low detection limit $(<1 \mathrm{mg}$ ), and excellent durability (reciprocating stretch and release $>1400$ times at strain 150\%). The self-similar Poly(vinylidenefluoride) (PVDF) nano/microfibers were fabricated by a newly-proposed helix electrohydrodynamic printing (HE-Printing) technique in combination with controllable in-surface self-organized buckling, in a low-cost, large-scale and aligned manner. It can individually manipulate the fiber flying in a helical manner, different from disordered dance of electrospinning and straight line of near-field electrospinning and mechanoelectrospinning [25]. Comprehensive experimental and theoretical studies proved that the HE-Printing technique can directly fabricate various serpentine nano/microfibers. The HSS, unlike filmbased sensors, never needs freestanding status to improve stretchability and avoid generating catastrophic out-of-surface deformation [34]. It showed repeatable and consistent electrical outputs with detection limit an order of magnitude smaller than the other stretchable sensors. Besides, it could simultaneously measure the own status and multiply extra mechanical stimuli such as the amplitude and speed of external loading, and keep stable sensing even under different stretching. The HSS was very high sensitive and utilized to remotely detect slight human motion including breathing and walking.

\section{Experimental section}

\subsection{Preparation of PVDF solution and substrates}

The PVDF (Kynar 761) was purchased from Arkema Investment Co. Ltd. N,N-dimethylformamide (DMF) and acetone were purchased from Sinopharm Chemical Reagent Co., Ltd. All the materials were used directly without further purification. The polymer PVDF (18 wt\%) was added in the solvent mixture DMF/acetone (50/50 wt\%), and heated at $35^{\circ} \mathrm{C}$ for $6 \mathrm{~h}$ to make the solution homogeneous.

Two kinds of substrates were adopted, Ecoflex substrate with microchannels and PDMS-on-Ecoflex substrate with excellent stickiness. 1) The Ecoflex solution (Ecoflex 0030, Smooth-On Inc.) was prepared by mixing the base and the curing agent with a ratio of $1: 1$, and cast into mold with channel pattern before being placed in a vacuum oven to remove air bubbles, then the solution was thermally cured at $60{ }^{\circ} \mathrm{C}$ for 10 min 2) For PDMS-on-Ecoflex substrate, Ecoflex solution was spincoated on glass substrate, followed by thermal curing at $60{ }^{\circ} \mathrm{C}$ for $10 \mathrm{~min}$, and the thickness was $\sim 0.5 \mathrm{~mm}$. Then PDMS solution (sylgard 184, Dow Corning, Inc.) (mixing the base and the curing agent with a ratio of 10:1) was spin-coated on the Ecoflex substrate and the PDMS layer's thickness is about $0.1 \mathrm{~mm}$. The mixture was first placed in a vacuum oven to remove air bubbles and then thermally cured at $60{ }^{\circ} \mathrm{C}$ for $40 \mathrm{~min}$ to obtain a half cured PDMS layer.

\subsection{Fabrication of self-similar fiber-based sensor}

The HE-Printing was utilized to fabricate piezoelectric PVDF nano/ microfibers. The PVDF solution was delivered using a syringe pump. A stainless-steel needle (inner and external diameter of $260 \mu \mathrm{m}$ and $510 \mu \mathrm{m}$ respectively) was adopted. A high voltage was exerted between nozzle and collector to generate jetting fiber through a power supplier (DW-P403, Dongwen Inc.).

Bidirectionally stretch the PDMS-on-Ecoflex substrate and place it onto silicon substrate with the PVDF nano/microfibers for several minutes, then quickly peel off and slowly release the elastomer to obtain self-similar nano/microfibers. Bond the Ecoflex substrate onto the PDMS-on-Ecoflex substrate with PVDF nano/microfibers to form microstructured channels, followed by injecting liquid metal (EgaIn, 75\% gallium and 25\% indium) to form stretchable interdigital electrodes. One needs to increase the stickiness between fibers and PDMS surface,
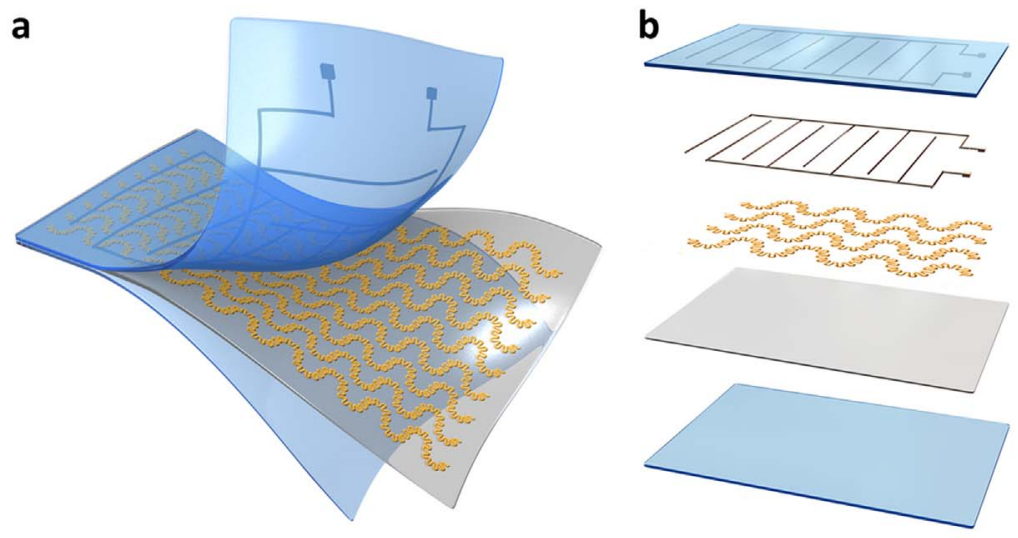

Ecoflex substrate
with channels
(Thickness $500 \mu \mathrm{m})$
Liquid metal
electrodes
Self-similar
PVDF fibers
PDMS layer
(Thickness $100 \mu \mathrm{m})$
Ecoflex substrate
(Thickness $500 \mu \mathrm{m})$


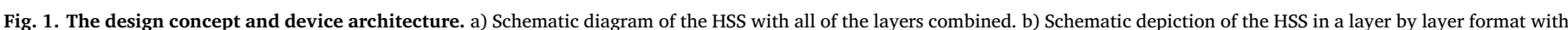

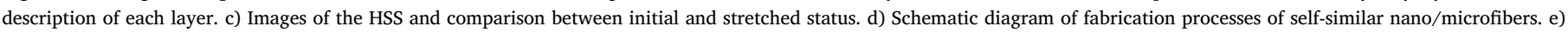
LSCM images of a self-similar nano/microfiber and SEM image of cross-section of the nano/microfiber. 
and reduce stickiness between fibers and silicon surface: 1) silane blocking agent was adopted to achieve hydrophobic treatment of silicon substrate, and 2) semi-solidified PDMS-on-Ecoflex substrate was utilized to receive nano/microfibers from silicon substrate.

\subsection{Characterization}

Infrared Radiation spectroscopy (IR) was performed with a Fourier transform infrared spectroscopy (FTIR) spectrophotometer (Spectrum 116 100, Perkin-Elmer Inc.), equipped with an IR grid polarizer (Specac Limited, UK), consisting of $0.12 \mathrm{~mm}$ wide strips of aluminium. A PANalytical X'pert MRD system, with $\mathrm{Cu}$ k-alpha radiation (wavelength $0.15418 \mathrm{~nm}$ ), crossed-slit collimator as primary optics, and a parallel plate collimator as secondary optics, a flat graphite monochromator and a proportional detector, was used for XRD measurements. The crystallinity of fibers was calculated from the area of diffraction peaks divided by the area of the whole diffraction curve. Supplementary Fig. S1a shows FTIR transmission spectrum of polarized fibers under various $U_{\text {applied. }}$ Polar $\beta$ phase bands appeared distinctly at 511, 600, 840 and $1275 \mathrm{~cm}^{-1}$, while the non-polar $\alpha$ phase bands were not obvious (411, 795, $1402 \mathrm{~cm}^{-1}$ ) [35]. Supplementary Fig. S1b indicated that the concentration of $\beta$ phase $F(\beta)$ increased monotonously with $U_{\text {applied. }}$ Infrared spectroscopy absorption bands at 795 and $840 \mathrm{~cm}^{-1}$ were chosen to characterize $\alpha$ and $\beta$ phase. The relatively faction of $\beta$ phase was calculated by $F(\beta)=\frac{X_{\beta}}{X_{\alpha}+X_{\beta}}=\frac{A_{\beta}}{\left(K_{\beta} / K_{\alpha}\right) A_{\alpha}+A_{\beta}}[36]$, where, $A_{\alpha}$ and $A_{\beta}$ are the absorbance at 795 and $840 \mathrm{~cm}^{-1}$, respectively, $X_{\alpha}$ and $X_{\beta}$ are the degree of crystallinity of $\alpha$ and $\beta$ phases, and $\mathrm{K}_{\alpha}=6.1 \times 10^{4} \mathrm{~cm}^{2}$ / mol and $K_{\beta}=7.7 \times 10^{4} \mathrm{~cm}^{2} / \mathrm{mol}$ are the absorption coefficients at the respective wave number.

\section{Results and discussion}

\subsection{Architecture of an HSS and HE-Printing technique}

An HSS and its exploded view of multilayer construction layout are schematically illustrated in Fig. 1a and b. Specifically, the HSS is based on a sandwich-structured composite, including aligned self-similar nano/microfibers of piezoelectric PVDF and liquid-metal electrode embedded between two layers of different substrates. The HSS can undergo large stretchability such as $\sim 320 \%$ (stretched from $\sim 36 \mathrm{~mm}$ to $\sim 154 \mathrm{~mm}$ ) and still work very well, as shown in Fig. 1c. Liquid metal, injected into interdigital microchannel with cross-section of $400 \mu \mathrm{m} \times 400 \mu \mathrm{m}$ embedded in Ecoflex substrate, acts as stretchable electrodes that deform with substrate and provide the stretchability matching with self-similar nano/microfibers. The PDMS-on-Ecoflex substrate utilizes the surface stickiness of fresh PDMS to achieve high bonding strength with nano/microfibers, and the hyperelasticity of Ecoflex to improve the stretchability. Fig. 1d illustrates the fabrication principle of self-similar nano/microfibers. It is a 3-step fabrication process: 1) HE-Printing deposits the initial serpentine nano/microfibers with tunable wavelength of 200-2000 $\mu \mathrm{m}$ and amplitude of $0-500 \mu \mathrm{m}$ on silicon substrate. 2) The PDMS-on-Ecoflex substrate, stretched by uniaxial/biaxial prestrain $\varepsilon_{\text {prestrain }}$ much larger than the critical strain $\varepsilon_{\text {critical }}$ of nano/microfiber buckling, is pressed down upon the silicon substrate [37]. 3) The PDMS-on-Ecoflex substrate is peeled off, followed by releasing the prestrain for the self-organized buckling of serpentine nano/microfibers to generate a "spring-on-spring" self-similar serpentine geometry, as shown in Fig. 1e. All the self-similar nano/microfibers were bonded on the PDMS-on-Ecoflex substrate, followed by covering Ecoflex layer with microchannel for the liquid-metal electrode.

We developed an HE-Printing technique by controlling the "whipping/buckling" instability of the electrical-field-driven jetting nano/ microfiber. The helical nano/microfiber accumulated into cylinder in the case of stationary collector (Supplementary Fig. S2), or formed versatile serpentine structures via the linear movement of collector with a

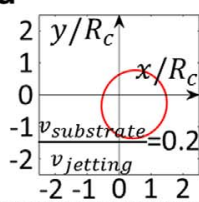

$\begin{array}{lllll}-2 & -1 & 0 & 1 & 2\end{array}$

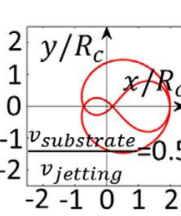

$\begin{array}{lllll}-2 & -1 & 0 & 1 & 2\end{array}$

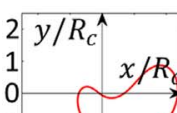

$-1 v_{\text {substrate }}=0.65$

-2 jetting

$\begin{array}{lllll}-2 & -1 & 0 & 1 & 2\end{array}$



2



$\begin{array}{lllllll}0 & 5 & 10 & 15 & 20 & 25 & 30\end{array}$
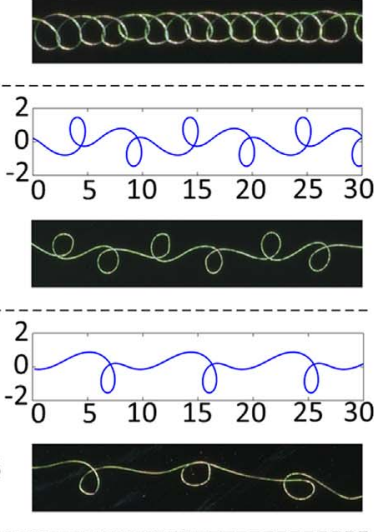

$-2$

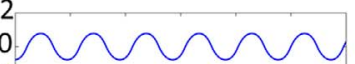

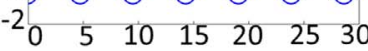

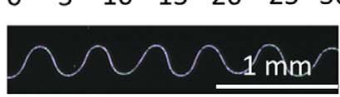


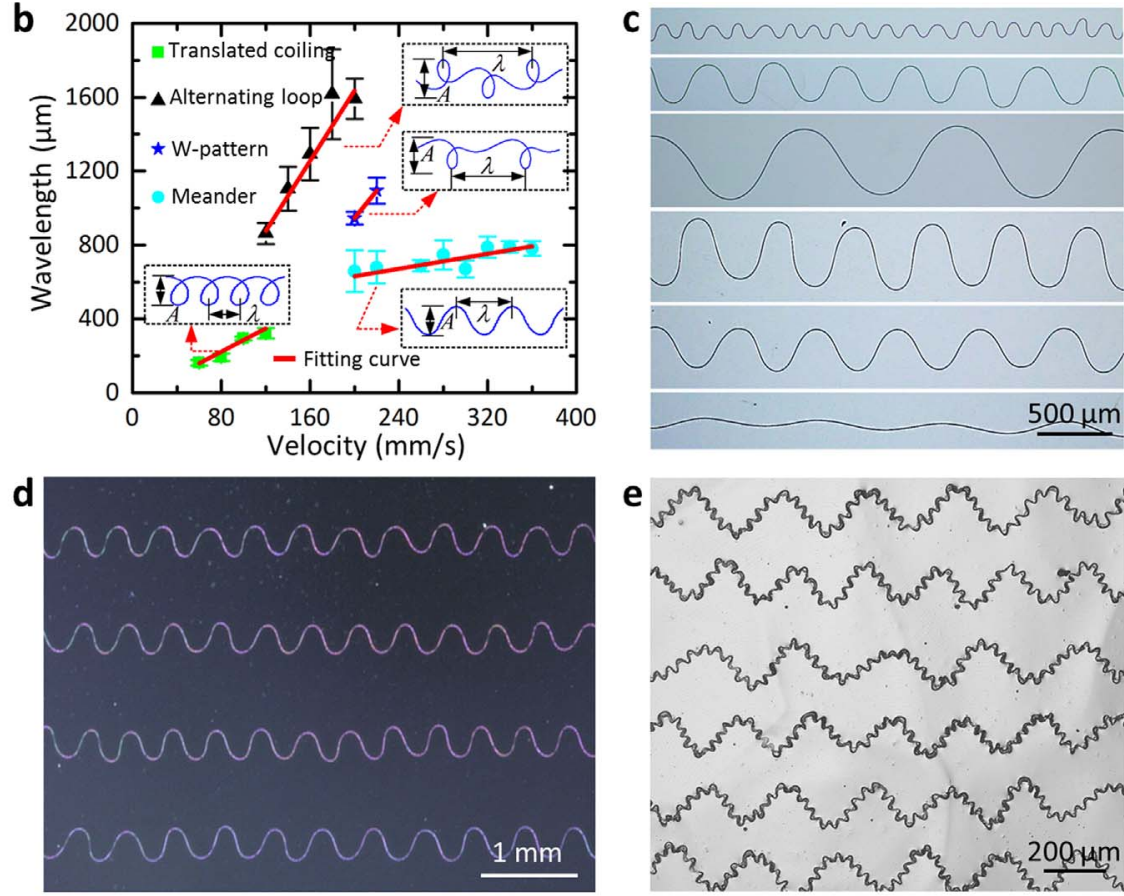

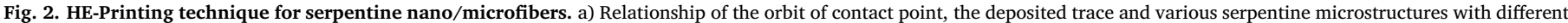

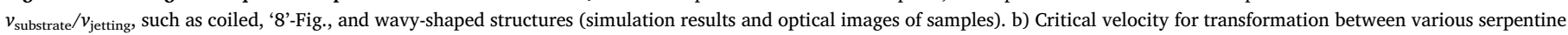

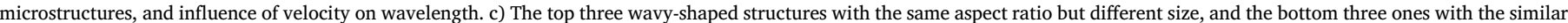

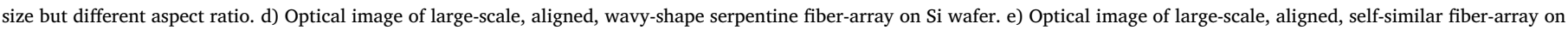
PDMS-on-Ecoflex substrate. 
different substrate speeds $v_{\text {substrate }}$ (Fig. 2a), where the applied voltage $U_{\text {applied }}$ was $1.5-3 \mathrm{kV}$ between a needle with inner diameter of $260 \mu \mathrm{m}$, fed by a syringe pump at a flow-rate $Q_{\text {flow }}$ of $300-600 \mathrm{nl} / \mathrm{min}$, and a collector at a distance of $h_{\text {nozzle }}$ from $10 \mathrm{~mm}$ to $35 \mathrm{~mm}$. The simulation of "whipping/buckling" instability agreed well with experimental observations (Supplementary Text 1), and give the velocity $v_{\text {jetting }}$ of jetting nano/microfiber at the contact point on silicon substrate.

Three key process parameters $\left(v_{\text {substrate, }}, U_{\text {applied, }}\right.$ and $h_{\text {nozzle }}$ ) can digitally manipulate the whipping nano/microfiber to form various geometries. 1) Tuning $v_{\text {substrate }}$ can generate four kinds of geometric patterns (coiled fibers, "8"-shaped fibers, wavy-shaped fibers and straight fibers). The laser scanning confocal microscope (LSCM) images in Fig. 2a showed the relationship of the orbit of contact point, the deposited trace and the patterns with the ratio $v_{\text {substrate }} / v_{\text {jetting }}$ by bifurcation theory of liquid ropes (discussed in Supplementary Text 2) [38]. Three critical substrate speeds, dividing four kinds of patterns, were $\sim 130 \mathrm{~mm} / \mathrm{s}, \sim 230 \mathrm{~mm} / \mathrm{s}$ and $\sim 360 \mathrm{~mm} / \mathrm{s}$, in the case of PVDF of $18 \%$ concentration, $U_{\text {applied }}=2.2 \mathrm{kV}, h_{\text {nozzle }}=25 \mathrm{~mm}$, and $Q_{\text {flow }}=$ $600 \mathrm{nl} / \mathrm{min}$. Additionally, $v_{\text {substrate }}$ controlled the wavelength and amplitude of serpentine geometry, as shown in Fig. 2b and Supplementary Fig. S4. 2) $U_{\text {applied }}$ simultaneously increased the jetting speed and helix rotation speed monotonously, and decreased the jetting diameter of deposited coil. 3) $h_{\text {nozzle }}$ was helpful for controlling the non-axisymmetric instability, and formed approximate circle cross-section for insurface buckling by tuning the solidification time of jetting. Various serpentine structural layouts were formed by digitally tuning the above process parameters, such as serpentine nano/microfibers with the same and various aspect ratios, and large-scale serpentine nano/microfibers, as shown in Fig. 2c and d.

\subsection{Fabrication of self-similar nano/microfibers}

The self-organized buckling of printed serpentine nano/microfibers represents an important approach to achieve excellent stretchability, where the prestrain $\varepsilon_{\text {prestrain }}$ is the driving force of buckling. $\varepsilon_{\text {prestrain, }}$ much larger than the in-surface critical strain $\varepsilon_{\text {critical-insurface }}$ of nano/ microfibers

$\varepsilon_{\text {critical-insurface }}=\frac{1}{\varphi} \sqrt{\frac{\bar{E}_{\mathrm{PDMS}}}{E_{\text {fiber }}}} \frac{\sqrt{I_{\text {fiber-insurface }}}}{A_{\text {fiber }}}$,

should be applied to the PDMS-on-Ecoflex substrate, where $E_{\text {fiber }}$ and $E_{\mathrm{PDMS}}$ are the Young's moduli of fiber and PDMS layer, $A_{\mathrm{fiber}}$ is the cross-section area of nano/microfiber, $I_{\text {fiber-insurface }}$ is the inertia moment of nano/microfiber in surface, $\varphi=\left(\frac{1}{2}-2 \gamma-2 \ln \left(\frac{5}{7}\right)-\frac{1}{2} \ln \left(\frac{W^{4} E_{\text {PDMS }}}{16 E_{\text {fiber }} I_{\text {fiber-insurface }}}\right)\right) \quad$ and $\quad \bar{E}_{\mathrm{PDMS}}=$ $E_{\mathrm{PDMS}} /\left(1-\nu_{\mathrm{PDMS}}^{2}\right), H$ and $W$ are the height and width of nano/microfiber cross-section [37]. We observed that the cross-section shape of nano/microfiber determines the buckling mode (in-/out-of-surface buckling), and the elastic modulus ratio $\bar{E}_{\mathrm{PDMS}} / E_{\text {fiber }}$ seriously influences the critical strains of in-surface and out-of-surface buckling modes, respectively, but exerts negligible influence on the transformation between buckling modes $[37,39]$. The critical aspect ratio of cross-section was predicted as $H / W \approx 0.9$ at $\bar{E}_{\mathrm{PDMS}} / E_{\mathrm{fiber}}=3.3 \times 10^{-4}$. So the nano/ microfibers with circle cross-section always buckle in surface, which was proved in our reported experiments [34].

Fig. 2e shows an LSCM image of large-scale, aligned, self-similar nano/microfibers self-organized from serpentine nano/microfibers of Fig. 2d. There were self-similar nano/microfibers with various sizes and similar aspect ratio and with various aspect ratios (Supplementary Fig. S5a and b). The initial serpentine geometry $\left(\lambda_{\text {initial }}\right.$ and $\left.A_{\text {initial }}\right)$ proportionally shrank to the first-level wavy geometry $\left(\lambda_{\text {level1 }}\right.$ and $\left.A_{\text {level1 }}\right)$ of self-similar geometry with the second wavy geometry $\left(\lambda_{\text {level2 }}\right.$ and $\left.A_{\text {level2 }}\right)$, namely $\lambda_{\text {level1 }}=\lambda_{\text {initial }} /\left(1+\varepsilon_{\text {prestrain_x }}\right)$ and $A_{\text {level1 }}=A_{\text {initial }} /$ $\left(1+\varepsilon_{\text {prestrain_y }}\right)$, where $\varepsilon_{\text {prestrain_x }}$ and $\varepsilon_{\text {prestrain_y }}$ are the prestrains along the direction of wavelength and amplitude. $\lambda_{\text {level2 }}$ and $A_{\text {level2 }}$ are $\lambda_{\text {level } 2}=\frac{14 \pi}{5}\left(\frac{E_{\text {fiber }} \text { Iin-surface }_{\text {PDMS }}}{E_{\text {PDS }}}\right)^{\frac{1}{4}}$ and $A_{\text {level } 2}=\frac{\lambda_{\text {level } 2}}{\pi} \sqrt{\varepsilon_{\text {prestrain }}-\varepsilon_{\text {critical }}}$, respectively. The theoretical prediction of $\lambda_{\text {level2 } 2}$ is $59 \mu \mathrm{m}$, and agreed well with the finite element simulation (commercial software ABAQUS) ( $55 \mu \mathrm{m})$ and experiment $(\sim 50 \mu \mathrm{m})$, where the diameter of nano/microfibers was $\sim 2 \mu \mathrm{m}, E_{\text {fiber }}=2 \mathrm{GPa}, v_{\text {fiber }}=0.3, E_{\mathrm{PDMS}}=1 \mathrm{MPa}$ and $\nu_{\mathrm{PDMS}}=0.45$. It was observed that the second-order serpentine shape was about one-order of magnitude smaller than the initial one. Additionally, the experimental results of Supplementary Fig. S5c show that the prestrain decreased the size of the first-level wavy geometry, and increased the density of the second-level wavy geometry, where the prestrains were $30-120 \%$. The prestrain improved the stretchability of serpentine nano/microfibers with the increasement of prestrain, which was proved by the finite element simulation, as shown in Supplementary Fig. S5d. Similarly, the applied strains $\varepsilon_{\text {applied }}$ also changed the shape of self-similar serpentine nano/microfibers, where $\varepsilon_{\text {applied }}=0 \%, 45 \%$ and $80 \%$, as shown in Supplementary Fig. S6.

The amplitude and direction of biaxial prestrain were used to tune the geometric shape of self-similar nano/microfibers. Importantly, the direction of prestrain was capable of controlling the buckling region of the serpentine nano/microfiber. For example, when releasing $\varepsilon_{\text {prestrain_x }}$, the second-level wavy appeared on the whole serpentine nano/microfiber with small aspect ratio uniformly, and merely at the peaks and valleys of large aspect-ratio serpentine nano/microfiber merely (Supplementary Fig. S7a). Suppose that the serpentine geometry is made up of a series of gradient line segments. Supplementary Fig. S7b presented the effect of direction of prestrain on the wavelength and amplitude, agreeing very well with the simulation of Supplementary Fig. S7c (the angles are $0^{\circ}, 30^{\circ}, 45^{\circ}, 60^{\circ}$, and $90^{\circ}$ ). There is a critical angle between the prestrain and nano/microfiber, only below which the buckling appears. And Supplementary Fig. S7d experimentally showed the critical angle was $\sim 55^{\circ}$, which agree well with the theoretical prediction (Supplementary Text 3.1)

$\theta_{1}=\tan ^{-1}\left(\frac{\sin \left(\theta_{0}\right)}{1-\nu_{\mathrm{PDMS}} \varepsilon_{\text {prestrain }}} / \frac{\cos \left(\theta_{0}\right)}{1+\varepsilon_{\text {prestrain }}}\right)=55^{\circ}$,

where, $\theta_{0}$ was resulted from $\frac{L_{1}}{L_{0}}=\sqrt{\left(\frac{\sin \left(\theta_{0}\right)}{1+\varepsilon_{\text {prestrain }}}\right)^{2}+\left(\frac{\cos \left(\theta_{0}\right)}{1-\nu_{\text {PDMS }} \varepsilon_{\text {prestrain }}}\right)^{2}}=1-$ $\varepsilon_{\text {critical }}$, and $\theta_{0}, \theta_{1}, L_{0}$ and $L_{1}$ are the angle and length before and after releasing the prestrain, respectively (Supplementary Fig. S8a). Supplementary Text 3.1 discussed the relationship of deformation between the nano/microfiber and substrate under uniaxial tensile. Here, we established a calculation law for sinusoidal geometry $\left(A_{\text {levelo }}\right.$ and $\left.\lambda_{\text {level0 }}\right)$ for uniform self-similar nano/microfiber. The critical aspect ratio $\beta_{\text {critical }}=\frac{A_{\text {level0 }}}{\lambda_{\text {level0 }}}=\frac{q\left(\varepsilon_{\text {prestrain }}\right)}{2 \pi \sqrt{1-q^{2}\left(\varepsilon_{\text {prestrain }}\right)}}$ is a prestrain-related function, and more details can be found in Supplementary Text 3.2. When the aspect ratio is smaller than $\beta_{\text {critical }}$ the self-similar nano/microfibers can be fabricated. Additionally, Supplementary Text 3.3 established the model under biaxial tensile condition, which demonstrated that the ratio of prestrains in two directions played a critical role in determining the critical angle for buckling. Interestingly, Supplementary Fig. S8c shows the inclined nano/microfibers underwent compression before stretching, and Fig. S8d explained the influence of the ratio of prestrains on buckling.

\subsection{Characterization of the HSS}

HE-Printing in situ electrically polarized PVDF nano/microfibers to transform the basic crystalline phases ( $\alpha$ and $\gamma$ ) into the polar $\beta$ phase, for the generation of piezoelectricity [27]. The polarization monotonically increased with applied voltage, and tended to converge when $U_{\text {applied }}>1.7 \mathrm{kV}$, as shown in Supplementary Fig. S1. The detail characterization between the process parameters and the piezoelectricity was discussed in the Experimental Section. Fig. 3a shows the HSS underwent uniaxial and biaxial stretching, curling and crumpling. Fig. 3b 
a

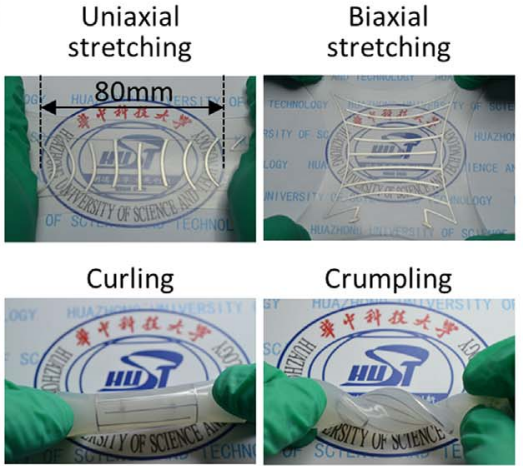

d

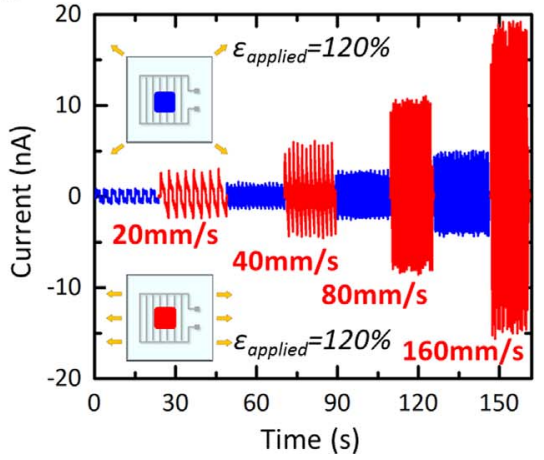

b

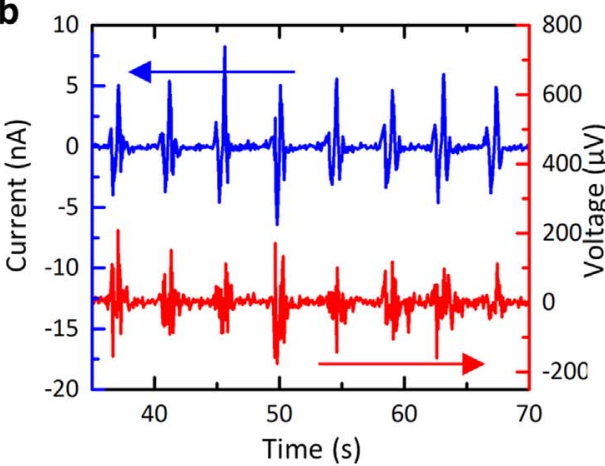

e

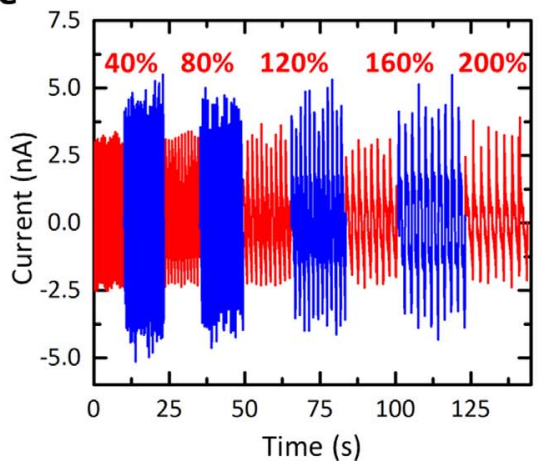



f

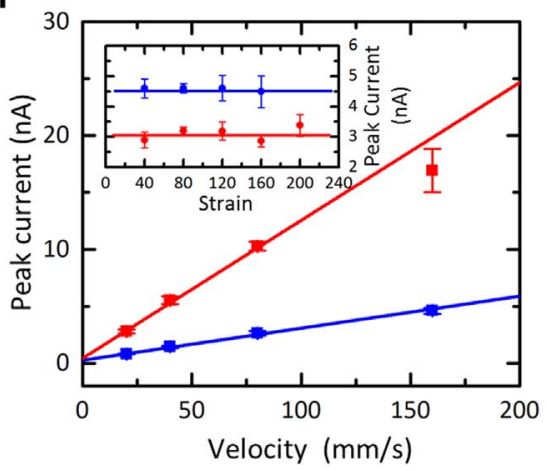

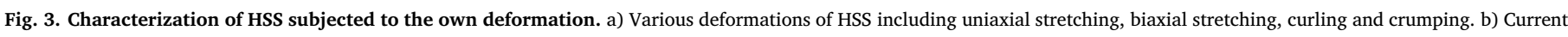

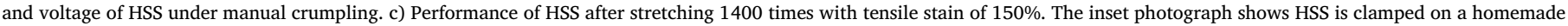

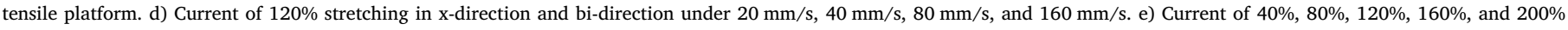
stretching in $\mathrm{x}$-direction and bi-direction, under the same velocity of $40 \mathrm{~mm} / \mathrm{s}$. f) Influence of amplitude and speed of stretching on peak current.

shows the current output and voltage output under arbitrary crumpling. In durability tests, the output kept stable even after more than 1400 times at the applied strain of $150 \%$, as shown in Fig. 3c, where the HSS was repeatedly stretched and released by a homemade automatic stretcher connected with the semiconductor analysis tester (Supplementary Movie S1).

Supplementary material related to this article can be found online at http://dx.doi.org/10.1016/j.nanoen.2017.07.048.

Fig. 3d shows the current output generated by the uniaxial and biaxial applied strains. The results in red color were the current from uniaxial stretching $120 \%$ along $\mathrm{x}$-direction with tensile speed of $20 \mathrm{~mm} / \mathrm{s}, 40 \mathrm{~mm} / \mathrm{s}, 80 \mathrm{~mm} / \mathrm{s}$, and $160 \mathrm{~mm} / \mathrm{s}$, and the peak current were $\sim 2.5 \mathrm{nA}, \sim 5 \mathrm{nA}, \sim 10 \mathrm{nA}$, and $\sim 20 \mathrm{nA}$, respectively. And the results in blue color were generated by biaxial stretching $120 \%$ with the same tensile load speeds of the red one. The figure declares that the outputs were a linear function of the stretching speed. Fig. 3e shows the outputs under uniaxial and biaxial stretching along x-direction with the uniform motion of $40 \mathrm{~mm} / \mathrm{s}$ of various strain amplitudes from $40 \%$ to $200 \%$ with an increment of $40 \%$. The maximum current approximatively kept constant for both uniaxial and biaxial stretching. And similar results under stretching can be obtained along y-direction (Supplementary Fig. S9a and b) and biaxial stretching. Fig. 3f plots the relationship of peak current change versus the tensile speed and amplitude of both uniaxial and biaxial stretching. It indicates that the current increased linearly with the tensile speed, and was free from the stretching amplitude. Based on the conclusion, the HSS can be utilized to measure the velocity of movement, without the influence of motion range, which will be further discussed in the next section. Supplementary Fig. S9c and d show the electrical output under bending by compression in $\mathrm{x}$-direction and y-direction with different compression deformations, at the tensile speed of $40 \mathrm{~mm} / \mathrm{s}$. The results indicate that the output was similar by bending with the same speed, even at different strain levels. The HSS can generate electricity under arbitrary twisting (Supplementary Fig. S9e).

\subsection{Applications of the HSS}

The ability to simultaneously measure the internal status and multiply external mechanical stimuli, such as the lateral pressure, the loading rate and the applied strains of substrate, is crucial for HSSs, which remains a challenge $[9,40]$. Fig. 4 shows the measurements of the amplitude (pressure or displacement) and speed (velocity or frequency) of external mechanical stimuli through HSSs in attached and freestanding modes. Fig. $4 \mathrm{a}$ is the homemade loading platform, based on CNC-MLM (computer numerical control multi-linkage mechanism). The applied load is calculated by using the Hooke's law and the deformation of PDMS pad (controlled by CNC-MLM). Fig. 4b shows the current of applied loads with different speeds (frequencies are $1.0 \mathrm{~Hz}$, $1.3 \mathrm{~Hz}$ and $1.6 \mathrm{~Hz}$ ) and the same amplitude ( $2 \mathrm{~mm}$ in experiment) of mechanical stimuli on the covered metal pad. The positive current output was resulted from the springback of soft substrate, which was used to measure the amplitude of mechanical stimuli. The negative current output is a linear increasing function of the speed of mechanical stimuli. The current output is consistent with fundamental piezoelectric theory $i_{\text {fiber }}=d_{33} E_{\text {fiber }} A_{\text {fiber }} \varepsilon_{\text {applied }}[41,42]$, where $i_{\text {fiber }}$ is the current output, $d_{33}$ is the piezoelectric charge constant, and $\varepsilon_{\text {applied }}^{\prime}$ is the applied strain rate (proportional to the speed of mechanical stimuli). Conversely, Fig. 4c shows the results of mechanical stimuli with the same speed $(1.6 \mathrm{~Hz})$ and different amplitudes $(0.8 \mathrm{~mm}, 1.4 \mathrm{~mm}$ and $2 \mathrm{~mm}$ ). The positive output linearly increases with the amplitude, and the negative output can remain the same due to the same speed. It indicates that the HSS can simultaneously and directly measure the amplitude and speed of mechanical stimuli, based on the positive current and negative current. 

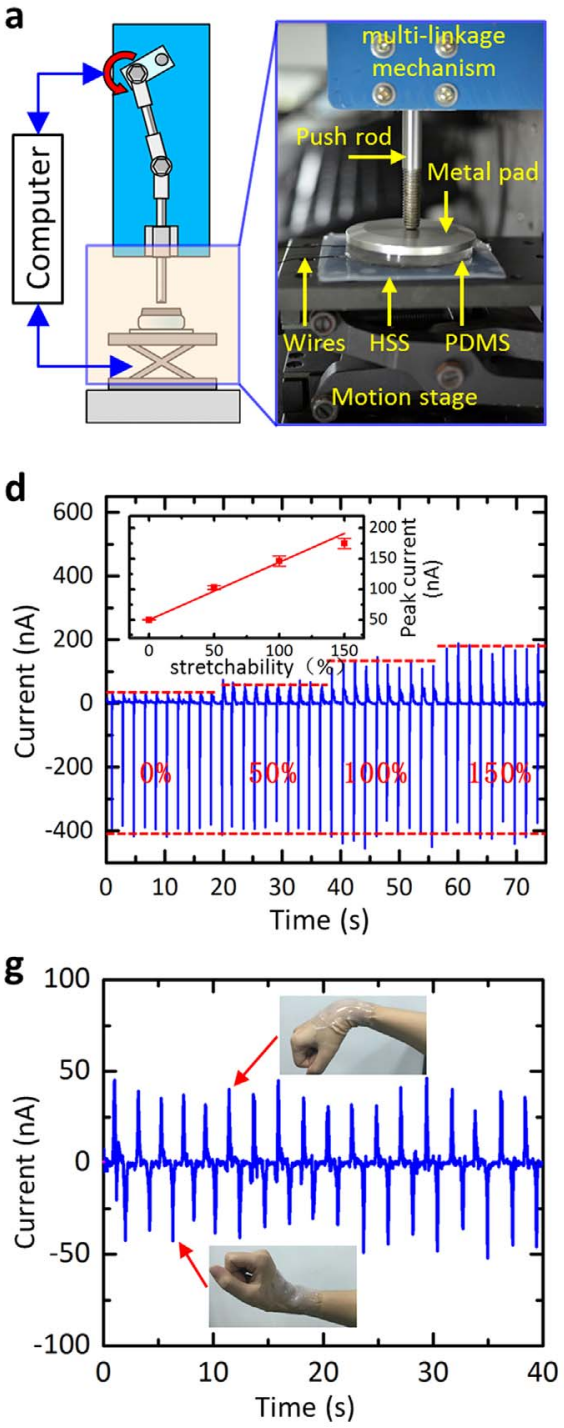
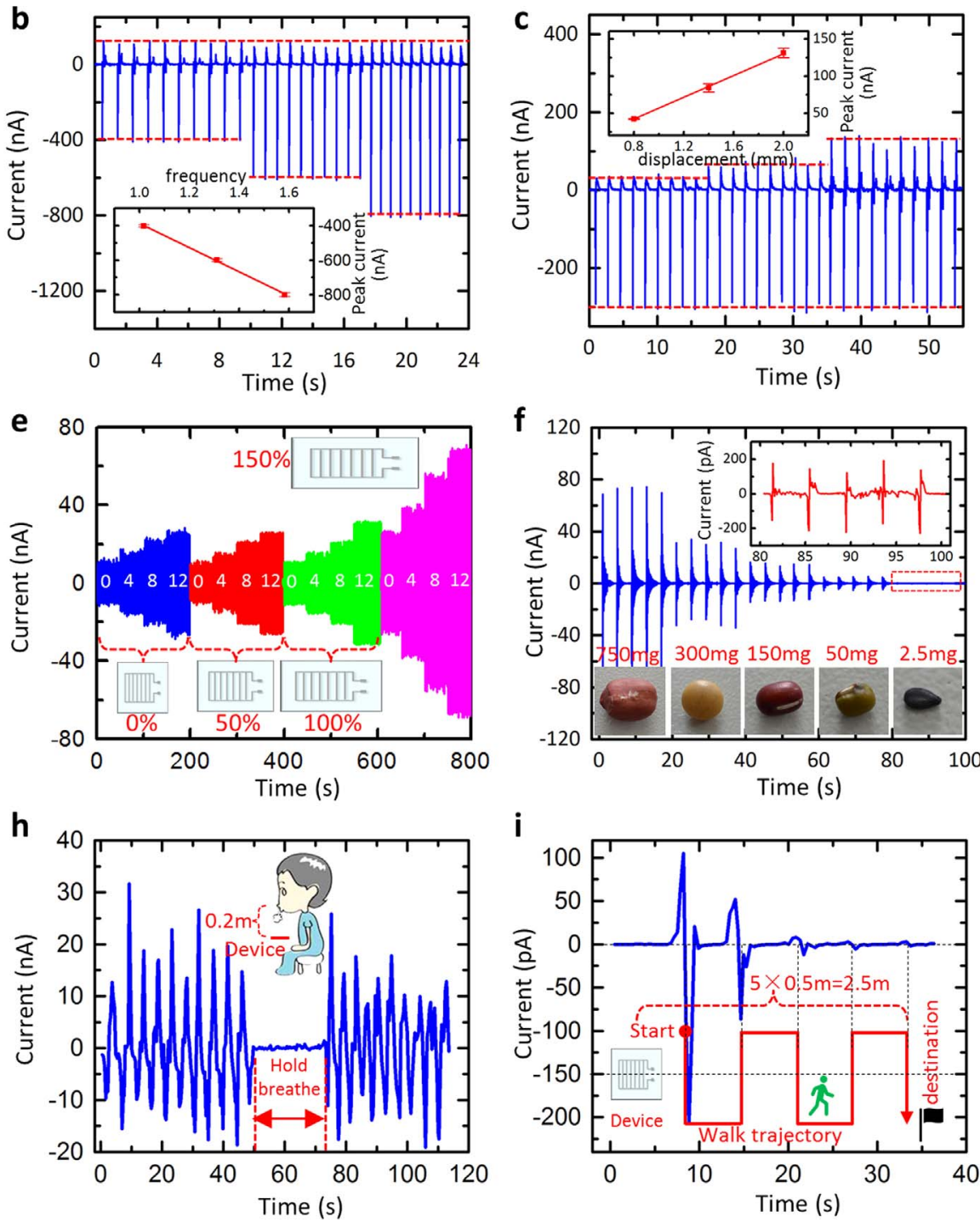

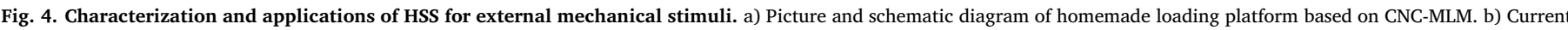



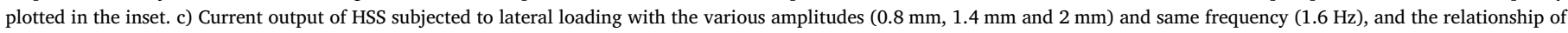

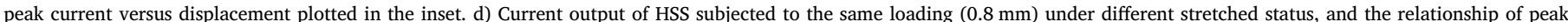





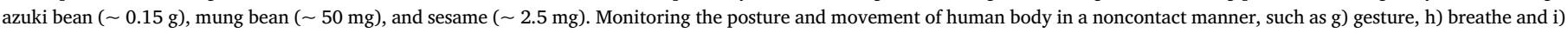
walking.

Supplementary material related to this article can be found online at http://dx.doi.org/10.1016/j.nanoen.2017.07.048.

Besides, the HSS should never decrease their sensitivity under large stretched state. Fig. $4 \mathrm{~d}$ shows the output results of the HSS stretched by various applied strains and subjected to the same loading $(0.8 \mathrm{~mm})$ in the case of attachment mode. The bottom constant output shows the adoption of the same amplitude of mechanical stimuli, and the peak current denotes the amplitude of mechanical stimuli. However, the upper output linearly increases with the applied strain. The main reason is that the applied strain decreases the thickness and increases the stiffness of the HSS. It improves the springback speed, and thereby enlarges the current. It proved that the HSS not only measured the lateral pressure, but also itself stretched strain. Fig. 4e shows the output results of the HSS under various lateral loadings $(0 \mathrm{~mm}, 4 \mathrm{~mm}, 8 \mathrm{~mm}$ and $12 \mathrm{~mm})$ and applied strains $(0 \%, 50 \%, 100 \%$ and $150 \%)$ in freestanding status. The output current was symmetric, different from those asymmetric outputs in the attachment mode. The results indicated that the current was mainly determined by the lateral pressure when $\varepsilon_{\text {applied }}<100 \%$. The current significantly increased when the HSS were stretched up to $150 \%$. So the HSS can be utilized to measure the lateral pressure under $\varepsilon_{\text {applied }}<100 \%$, and can give warning when the HSS stretched beyond the threshold such as $150 \%$. Further, to study the detection limit, the HSS were utilized to sense various small objects. Fig. 4f shows the measurement of falling of different grains in freestanding status. The falling down from $10 \mathrm{~cm}$ of nearly spherical grains, including peanut $(\sim 0.75 \mathrm{~g})$, soybean $(\sim 0.3 \mathrm{~g})$, azuki bean $(\sim 0.15 \mathrm{~g})$, mung bean $(\sim 50 \mathrm{mg})$ and sesame $(\sim 2.5 \mathrm{mg})$, was adopted to exhibit the excellent sensing performance. The impact loads, calculated by $F=\sqrt{2 g \cdot x} \cdot m / t$, for the five objects are $13.1 \times 10^{-3} \mathrm{~N}, 7 \times 10^{-3} \mathrm{~N}$, $4.67 \times 10^{-3} \mathrm{~N}, 1.55 \times 10^{-3} \mathrm{~N}$ and $2.92 \times 10^{-5} \mathrm{~N}$, respectively, where $g$ is the gravitational acceleration, $x$ is the falling distance, $m$ is the mass of the object, and $t$ is the impact time $(0.06 \mathrm{~s}-0.12 \mathrm{~s}$ obtained from Fig. 4f). The detail of experiment can be observed clearly in Supplementary Movie S2.The HSS also can detect as small as $0.2 \mathrm{mg}$ objects such as paper with $1 \mathrm{~mm} \times 1 \mathrm{~mm}$ as shown in Supplementary 
Fig. S10,

The HSS has potential applications in monitoring the posture and movement of human body. The HSS was attached on wrist comfortably using scotch tape at sides. Fig. $4 \mathrm{~g}$ shows the output current changed with motion of wrist, based on which the wrist configuration was assessed. It was also used for sensing touching under different stretched status, as shown in Supplementary Fig. S9f. The electrical output from the finger tapping under the strains from $0 \%$ to $200 \%$ with increment of $40 \%$. It proved that the stretching of HSS was beneficial in increasing the sensitivity. Additionally, the HSS could detect very slight human motions in a noncontact manner. When the sensor was placed not far from the nose, such as $20 \mathrm{~cm}$, the breathing rate was monitored (Fig. 4h). Fig. 4i shows the electrical signals appeared when human walked through HSS, as shown in Supplementary Movie S3. The peak current decreased with the increasing of distance between the HSS and human, since the air disturbance from human motion becomes weak with the increasing of distance. The HSS can monitor the walking steps and distance of human.

Supplementary material related to this article can be found online at http://dx.doi.org/10.1016/j.nanoen.2017.07.048.

\section{Conclusions}

In summary, we proposed a hyper-stretchable self-powered sensor (HSS) based on electrohydrodynamically printed, self-similar nano/ microfibers and embedded liquid-metal electrodes. The HSS showed high stretchability (up to $320 \%$ ), low detection limit $(0.2 \mathrm{mg}$ ) and excellent stability under reciprocating deformation tests (1400 times at stretching 150\%). A new HE-Printing technique was introduced for versatile and rapid fabrication of serpentine/self-similar fiber-based structures of piezoelectric polymeric materials, in a low-cost, largescale and aligned manner. The HSS adopted a 'self-similar' design that offers unique, 'spring-on-spring' structures for enhanced stretchability, which is the magnitude of prestrain larger than the initial serpentine nano/microfibers. Experimental and theoretical studies provided guidelines for the design, fabrication and applications of the HSS. Significantly, the HSS showed high sensitivity up to detecting $0.2 \mathrm{mg}$ and multifunctional ability to simultaneously measure their own status and multiply extra mechanical stimuli. The HSS avoid abrupt change of measurement resulted from inevitable catastrophic collapse of out-ofsurface buckling deformation of film-based sensors. We envision that the HSS with high stretchability and high sensitivity has potential applications in super artificial skin for humanoid robotics, human-machine interfaces, and physiological analysis devices.

\section{Acknowledgments}

The authors acknowledge supports from the National Natural Science Foundation of China $(51635007,91323303)$ and The National Key Research and Development Program of China (2016YFB0401105). The general characterization facilities are provided by the Flexible Electronics Manufacturing Laboratory in Comprehensive Experiment Center for Advanced Manufacturing Equipment and Technology at Huazhong University of Science and Technology.

\section{Appendix A. Supporting information}

Supplementary data associated with this article can be found in the online version at http://dx.doi.org/10.1016/j.nanoen.2017.07.048.
[3] D.H. Kim, N.S. Lu, R. Ma, Y.S. Kim, R.H. Kim, S.D. Wang, J. Wu, S.M. Won, H. Tao, A. Islam, K.J. Yu, T.I. Kim, R. Chowdhury, M. Ying, L.Z. Xu, M. Li, H.J. Chung, H. Keum, M. McCormick, P. Liu, Y.W. Zhang, F.G. Omenetto, Y.G. Huang, T. Coleman, J.A. Rogers, Science 333 (2011) 838-843.

[4] T. Huang, C. Wang, H. Yu, H.Z. Wang, Q.H. Zhang, M.F. Zhu, Nano Energy 14 (2015) 226-235.

[5] H. Wu, Y. Huang, F. Xu, Y. Duan, Z. Yin, Adv. Mater. 28 (2016) 9881-9919.

[6] J. Chen, G. Zhu, J. Yang, Q.S. Jing, P. Bai, W.Q. Yang, X.W. Qi, Y.J. Su, Z.L. Wang, ACS Nano 9 (2015) 105-116.

[7] J.W. Jeong, W.H. Yeo, A. Akhtar, J.J.S. Norton, Y.J. Kwack, S. Li, S.Y. Jung, Y.W. Su, W. Lee, J. Xia, H.Y. Cheng, Y.G. Huang, W.S. Choi, T. Bretl, J.A. Rogers, Adv. Mater. 25 (2013) 6839-6846.

[8] G. Schwartz, B.C.K. Tee, J.G. Mei, A.L. Appleton, D.H. Kim, H.L. Wang, Z.N. Bao, Nat. Commun. 4 (2013) 1859.

[9] J. Ge, L. Sun, F.R. Zhang, Y. Zhang, L.A. Shi, H.Y. Zhao, H.W. Zhu, H.L. Jiang, S.H. Yu, Adv. Mater. 28 (2016) 722-728.

[10] H.X. He, Y.M. Fu, W.L. Zang, Q. Wang, L.L. Xing, Y. Zhang, X.Y. Xue, Nano Energy 31 (2017) 37-48.

[11] M.Y. Ma, Z. Zhang, Q.L. Liao, F. Yi, L.H. Han, G.J. Zhang, S. Liu, X.Q. Liao, Y. Zhang, Nano Energy 32 (2017) 389-396.

[12] Z. Lou, S. Chen, L. Wang, R. Shi, L. Li, K. Jiang, D. Chen, G. Shen, Nano Energy 38 (2017) 28-35.

[13] T. Yamada, Y. Hayamizu, Y. Yamamoto, Y. Yomogida, A. Izadi-Najafabadi, D.N. Futaba, K. Hata, Nat. Nanotechnol. 6 (2011) 296-301.

[14] S. Lim, D. Son, J. Kim, Y.B. Lee, J.K. Song, S. Choi, D.J. Lee, J.H. Kim, M. Lee, T. Hyeon, Adv. Funct. Mater. 25 (2015) 375-383.

[15] X.B. Zang, M. Zhu, X. Li, X.M. Li, Z. Zhen, J.C. Lao, K.L. Wang, F.Y. Kang, B.Q. Wei, H.W. Zhu, Nano Energy 15 (2015) 83-91.

[16] Y.J. Fan, X.S. Meng, H.Y. Li, S.Y. Kuang, L. Zhang, Y. Wu, Z.L. Wang, G. Zhu, Adv. Mater. 29 (2017) 1603115.

[17] T. Sekitani, Y. Noguchi, K. Hata, T. Fukushima, T. Aida, T. Someya, Science 321 (2008) 1468-1472.

[18] F. Yi, X.F. Wang, S.M. Niu, S.M. Li, Y.J. Yin, K.R. Dai, G.J. Zhang, L. Lin, Z. Wen, H.Y. Guo, J. Wang, M.H. Yeh, Y.L. Zi, Q.L. Liao, Z. You, Y. Zhang, Z.L. Wang, Sci. Adv. 2 (2016) e1501624.

[19] Y.A. Huang, Y.Z. Wang, L. Xiao, H.M. Liu, W.T. Dong, Z.P. Yin, Lab Chip 14 (2014) 4205-4212.

[20] C.S. Wu, X. Wang, L. Lin, H.Y. Guo, Z.L. Wang, ACS Nano 10 (2016) 4652-4659.

[21] Y. Huang, W. Dong, T. Huang, Y. Wang, L. Xiao, Y. Su, Z. Yin, Sens. Actuators A: Phys. 224 (2015) 36-42.

[22] Y. Su, S. Wang, Y. Huang, H. Luan, W. Dong, J.A. Fan, Q. Yang, J.A. Rogers, Y. Huang, Small 11 (2015) 367-373.

[23] J. Yun, Y. Lim, G.N. Jang, D. Kim, S.J. Lee, H. Park, S.Y. Hong, G. Lee, G. Zi, J.S. Ha, Nano Energy 19 (2016) 401-414.

[24] X. Wang, Y. Yin, F. Yi, K. Dai, S. Niu, Y. Han, Y. Zhang, Z. You, Nano Energy 39 (2017) 429-436.

[25] Y.K. Fuh, P.C. Chen, Z.M. Huang, H.C. Ho, Nano Energy 11 (2015) 671-677.

[26] L. Persano, C. Dagdeviren, Y. Su, Y. Zhang, S. Girardo, D. Pisignano, Y. Huang, J.A. Rogers, Nat. Commun. 4 (2013) 1633.

[27] C.E. Chang, V.H. Tran, J.B. Wang, Y.K. Fuh, L.W. Lin, Nano Lett. 10 (2010) 726-731.

[28] S.C.B. Mannsfeld, B.C.K. Tee, R.M. Stoltenberg, C.V.H.H. Chen, S. Barman, B.V.O. Muir, A.N. Sokolov, C. Reese, Z. Bao, Nat. Mater. 9 (2010) 859-864.

[29] X. Huang, Y. Liu, K. Chen, W.J. Shin, C.J. Lu, G.W. Kong, D. Patnaik, S.H. Lee, J.F. Cortes, J.A. Rogers, Small 10 (2014) 3083-3090.

[30] G. Zhu, W.Q. Yang, T. Zhang, Q. Jing, J. Chen, Y.S. Zhou, P. Bai, Z.L. Wang, Nano Lett. 14 (2014) 3208-3213.

[31] Y.S. Kim, Y.N. Xie, X.N. Wen, S.H. Wang, S.J. Kim, H.K. Song, Z.L. Wang, Nano Energy 14 (2015) 77-86.

[32] C. Dagdeviren, Y. Shi, P. Joe, R. Ghaffari, G. Balooch, K. Usgaonkar, O. Gur, P.L. Tran, J.R. Crosby, M. Meyer, Y.W. Su, R.C. Webb, A.S. Tedesco, M.J. Slepian, Y.G. Huang, J.A. Rogers, Nat. Mater. 14 (2015) 728-+.

[33] J.W. Zhong, Q.Z. Zhong, Q.Y. Hu, N. Wu, W.B. Li, B. Wang, B. Hu, J. Zhou, Adv. Funct. Mater. 25 (2015) 1798-1803.

[34] Y. Duan, Y. Huang, Z. Yin, N. Bu, W. Dong, Nanoscale 6 (2014) 3289-3295.

[35] J.S. Andrew, D.R. Clarke, Langmuir 24 (2008) 670-672.

[36] M.M.D. Ramos, H.M.G. Correia, S. Lanceros-Mendez, Comput. Mater. Sci. 33 (2005) 230-236.

[37] Y.Q. Duan, Y.A. Huang, Z.P. Yin, J. Phys. D.-Appl. Phys. 48 (2015) 045302.

[38] P.T. Brun, B. Audoly, N.M. Ribe, T.S. Eaves, J.R. Lister, Phys. Rev. Lett. 114 (2015) 174501.

[39] Y.A. Huang, Y.Q. Duan, Y.J. Ding, N.B. Bu, Y.Q. Pan, N.S. Lu, Z.P. Yin, Sci. Rep. 4 (2014) 5949.

[40] S. Park, H. Kim, M. Vosgueritchian, S. Cheon, H. Kim, J.H. Koo, T.R. Kim, S. Lee, G. Schwartz, H. Chang, Z.A. Bao, Adv. Mater. 26 (2014) 7324-7332.

[41] J. Sirohi, I. Chopra, J. Intell. Mater. Syst. Struct. 11 (2000) 246-257.

[42] Y.J. Ding, Y.Q. Duan, Y.A. Huang, Energy Technol.-Ger. 3 (2015) 351-358.

\section{References}

[1] X. Pu, M. Liu, X. Chen, J. Sun, C. Du, Y. Zhang, J. Zhai, W. Hu, Z.L. Wang, Sci. Adv. 3 (2017) e1700015.

[2] X. He, Y.L. Zi, H.Y. Guo, H.W. Zheng, Y. Xi, C.S. Wu, J. Wang, W. Zhang, C.H. Lu, Z.L. Wang, Adv. Funct. Mater. 27 (2017) 201604378. 




YongAn Huang received the B.S. degree (2001), M.S. degree (2004) and Ph.D. degree (2007) from the Northwestern Polytechnical University, Xian, China. Since 2007, he has been a Faculty member at the State Key Laboratory of Digital Manufacturing Equipment and Technology, Huazhong University of Science and Technology (HUST), where he became a professor in 2014. His research interests include flexible electronics manufacturing and advanced printing.



Yajiang Ding received his B.S. degree from Huazhong University of Science and Technology (HUST) in 2013. He is now a Ph.D. candidate in the State Key Laboratory of Digital Manufacturing Equipment and Technology, School of Mechanical Science and Engineering, HUST. His research is focused on mechano-electrospinning and organic thinfilm transistor for flexible electronics manufacturing.

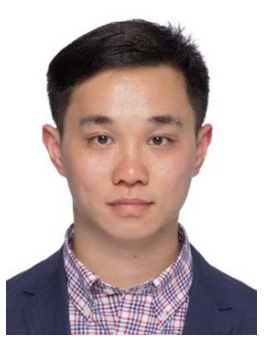

Jing Bian received his B.S. degree from Huazhong University of Science and Technology (HUST) in 2015. He is now a Ph.D. candidate in the State Key Laboratory of Digital Manufacturing Equipment and Technology, School of Mechanical Science and Engineering, HUST. His research is focused on laser lift-off technique for flexible electronics manufacturing.

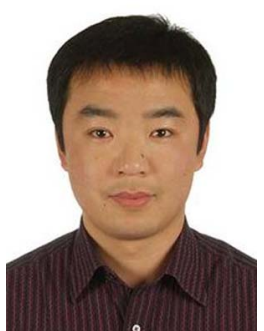

Yewang Su received the Ph.D. degree in engineering mechanics from Tsinghua University, Beijing, China, in 2011. He was a post-doctoral fellow with Departments of Civil and Environmental Engineering and Mechanical Engineering, Northwestern University, Evanston, IL, US, from 2011 to 2014. He is currently a research fellow in State Key Laboratory of Nonlinear Mechanics, Institute of Mechanics, Chinese Academy of Sciences, Beijing, China. His research interests include Gas shale, Stretchable electronics and Biomechanics.

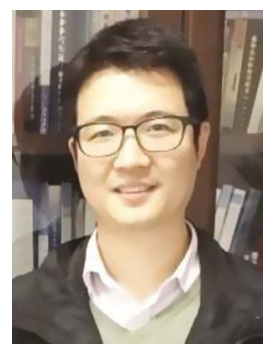

Jun Zhou received his B.S. degree in Material Physics (2001) and his Ph.D. in Material Physics and Chemistry (2007) from Sun Yat-Sen University, China. During 2005-2006, he was a visiting student in Georgia Institute of Technology. After he obtaining his Ph.D., He worked in Georgia Institute of Technology as a research scientist. He joined in Wuhan National Laboratory for Optoelectronics (WNOL), Huazhong University of Science and Technology (HUST) as a professor from the end of 2009. His main research interest is flexible energy harvesting and storage devices for self-powered micro/nanosensor systems.



Yongqing Duan received the B.S. and Ph.D. degrees from Huazhong University of Science and Technology (HUST), Wuhan, China, in 2010 and 2015 respectively. She is now a postdoctoral fellow in the State Key Laboratory of Digital Manufacturing Equipment and Technology, School of Mechanical Science and Engineering, HUST. Her research interests include flexible electronics manufacturing and electrohydrodynamic printing.

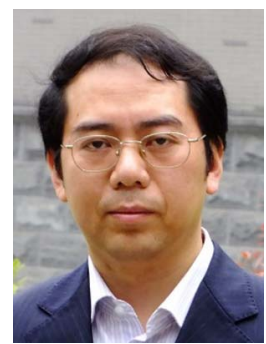

Zhouping Yin received the B.S. and Ph.D. degrees in mechanical engineering from Huazhong University of Science and Technology (HUST), Wuhan, China, in 1994 and 2000, respectively. He is a professor in school of mechanical science and engineering, HUST. He was awarded the China National Funds for Distinguished Young Scientists in 2006 He is a "Cheung Kong" Chair Professor of HUST since 2009. His research interests include electronic manufacturing equipment and technology, RFID technology and applications, digital manufacturing and applications. 